'Facultad de Ciencias Jurídicas y Sociales, Universidad de Talca. Santiago, Chile. ${ }^{2}$ Miembro Sociedad Chilena de Geriatría y Gerontología. ${ }^{3}$ Laboratorio de Neuropsicología y Neurociencias Clinicas (LANNEC), Programa de Fisiopatología-

ICBM, Departamento de Ciencias Neurológicas Oriente y Neurociencias. Facultad de Medicina. Universidad de Chile. Santiago, Chile. ${ }^{4}$ Centro de Gerociencias. Salud Mental y Metabolismo. Santiago, Chile.

${ }^{5}$ Clínica de Memoria y Neuropsiquiatría (CMYN), Servicio de Neurología. Hospital del Salvador y Facultad de Medicina, Universidad de Chile. Santiago, Chile. ${ }^{6}$ Servicio de Neurología, Departamento de Medicina, Clínica AlemanaUniversidad del Desarrollo. Santiago, Chile.

${ }^{7}$ Centro de Investigación Avanzada en Educación, Universidad de Chile, Santiago, Chile. ${ }^{8}$ Corporación Profesional de Alzheimer y Otras Demencias COPRAD Chile. aAbogado, PhD. ${ }^{b} \mathrm{MD}, \mathrm{PhD}$.

Financiamiento CONICYT/ FONDECYT regular/1140423; Programa Fondap Proyecto 15150012 \& Centro Basal para Centros de Excelencia, Proyecto FB 0003 de Programa Investigación Asociativa CONICYT. Programa Fondef ID16AM0006.

Recibido el 30 de mayo de 2016 , aceptado el 29 de noviembre de 2016.

Correspondencia a: Dra. Andrea Slachevsky andrea.slachevsky@uchile.cl

\section{¿Sé y puedo? Toma de decisión y consentimiento informado en los trastornos demenciantes: dilemas diagnósticos y jurídicos en Chile}

ANGELAARENAS MASSA ${ }^{1,2, a}$, ANDREA SLACHEVSKY CHONCHOL ${ }^{3-8, b}$

In Chile, more than 180 thousand people (1\% of the population) have some form of dementia. The figure should increase to approximately 600,000 (3\% of Chileans) by 2050. This disease poses major challenges to the society. One of them is the effective recognition of the autonomy and responsibility of the person living with this condition. This article aims to review the clinical assessment of competence, its agreement with the Chilean legal system and the challenges that the assessment of competence poses in clinical decision-making and the capacity of an individual make decisions, according to the new international obligations subscribed by Chile. It is concluded that inclusion is a pending challenge, reflected among other things, by the non-compliance with binding rules such as Article 12 of the Convention on the Rights of Persons with Disabilities, which affirms that persons with disabilities have the right to be recognized as a person everywhere, before the law.

(Rev Med Chile 2017; 145: 1312-1318)

Key words: Clinical Decision-Making; Dementia; Disability Evaluation; Informed Consent.

L as demencias constituyen un problema relevante de salud pública; constituyen una causa creciente de discapacidad, dependencia y muerte a nivel internacional ${ }^{1,2}$. En Chile, más de 180 mil personas ( $1 \%$ de los chilenos) presenta algún tipo de demencia, cifra que se proyecta en 600.000 personas aproximadamente $(3 \%$ de los chilenos) para el $2050^{3}$. Al igual que en otros trastornos neuropsiquiátricos, ocurren situaciones complejas tanto éticas como jurídicas, entre ellas la determinación de los niveles de autonomía para la toma de decisiones en múltiples dominios como las actividades de la vida diaria y las decisiones en materia de salud ${ }^{4,5}$.

La toma de decisión de personas con de- mencias plantea diversos desafíos, entre ellos, la evaluación clínica de la competencia, si la competencia desde el punto de visto clínico coincide con el sistema jurídico que regula la capacidad en Chile y si este sistema jurídico obedece a requerimientos internacionales de la Convención de Derechos de las Personas con Discapacidad (2006). Esta discusión es relevante considerando que el ordenamiento chileno emplea el vocablo demente como sinónimo de locura o trastorno de razón, lo que es anacrónico al uso médico del término. También, existe una creencia falsa en la población general y en los profesionales de salud, según la cual las personas con demencia carecen de capacidad de tomar decisiones. 
Este artículo revisa la evaluación clínica de la competencia, su coincidencia con el sistema jurídico chileno y los desafíos que plantean la competencia y la capacidad ante las nuevas obligaciones internacionales asumidas por Chile.

\section{La competencia en la toma de decisiones clínicas}

La competencia de toma de decisión en salud es un elemento central de la autonomía y junto a la responsabilidad, constituyen principios orientadores de la relación entre el paciente o usuario y el equipo sanitario. Técnicamente la Declaración de Bioética y Derechos Humanos (2006) define estos conceptos como la facultad de adoptar decisiones sobre la propia vida, asumiendo la responsabilidad de estas y respetando a los demás. Se considera la autonomía del sujeto imprescindible para la integración de procesos decisionales, tales como el consentimiento informado. El consentimiento (acuerdo de voluntades) no dice relación con la satisfacción narcisa y el autonomismo del paciente, sino con la realización de su bien terapéutico posible 6 . También se ocupa de la decisión libre e informada que pueda experimentar un paciente respecto de una decisión de salud que le afecta, es decir, ausente de presión o constricción externa ${ }^{7}$. Paralelamente, la psicología experimental, las neurociencias y la neurología cognitiva han contribuido a develar que la sola racionalidad no determina la toma de decisión humana ${ }^{8}$. Esta requiere la integración de información cognitiva, emocional y motivacional. La evaluación de la competencia en la toma de decisiones es un proceso complejo en personas con o sin deterioro cognitivo. Enfrentadas a tareas decisionales y de razonamiento las personas generalmente fallan en usar la lógica y las respuestas suelen basarse en intuiciones y corazonadas, que se puede manifestar en la presencia de algunos sesgos en las respuestas. ${ }^{9}$. Por un lado, lo descrito puede incidir en la comprensión de los consentimientos informados de personas sin deterioro ${ }^{10,11}$. Un ejemplo de ello es la asimetría en el tratamiento de las ganancias y pérdidas ${ }^{12}$ dado que existe una tendencia a sobreestimar la probabilidad de ocurrencia de eventos que deseamos y subestimar la de eventos indeseados ${ }^{13}$. Por otro lado, el deterioro cognitivo puede afectar o no la competencia de toma de decisión, requiriendo una evaluación ad hoc del proceso de formación de consentimiento.

Se considera que una persona es competente para tomar decisiones o realizar una actividad o en determinada materia, si sus capacidades o habilidades están sobre el nivel requerido para su realización ${ }^{14}$. La competencia es un concepto umbral. En términos prácticos, el umbral que se establece entre la competencia o incompetencia para decidir está determinado por las capacidades que permiten obtener un consentimiento informado ${ }^{15}$. Estas capacidades dependen de cuatro componentes fundamentales: 1) la capacidad de entender y recordar información; 2) la capacidad de manipular la información de manera critica; 3) la libertad de elegir y 4) la capacidad de expresarse ${ }^{16}$. No existe un umbral absoluto entre competencia e incompetencia ni un test consensuado para evaluar la capacidad de consentir. La confiabilidad y validez de tests, como "MacArthur Competence Assessment Tool for Clinical Research", "the California Scale of Appreciation" y el "Deaconess Informed Consent Comprehension Test" son limitadas y, no han sido validados en Chile ${ }^{15}$. En ausencia de instrumento universal para evaluar la capacidad de consentir, los profesionales de la salud e investigadores se basan en su juicio clínico e intuición ${ }^{14}$. Clarke (2013) propone que clínicos e investigadores fundamentan implícitamente su juicio en tres pilares: se presume que la mayoría de las personas sobre cierta edad (generalmente 14 años) pueden consentir ${ }^{15}$. Este criterio etario coincide con las normas jurídicas de distintos países. La competencia para consentir de una persona no varía, exceptuando cuando las personas están sometidas a circunstancias extremas y, si una persona es capaz de consentir a un procedimiento o tratamiento médico, es capaz de consentir a otros, independiente de su complejidad. Entonces, es necesario precisar cuando la intensidad del trastorno cognitivo interfiere con la capacidad o aptitud para tomar decisiones informadas y responsables en actividades de la vida diaria (manejar asuntos financieros, ejecutar algún deseo, manejar un vehículo, administrarse los medicamentos o vivir de forma independiente) y en asuntos de salud (aceptar o no una hospitalización o un procedimiento quirúrgico, dar consentimiento informado en una investigación científica $)^{14,17}$. La evaluación de competencia para consentir en personas con trastornos cognitivos presenta 
complejidades generales y particulares, en caso de trastornos demenciantes: No existe umbral absoluto para determinar la competencia de toma de decisión ni una relación lineal entre intensidad de las demencias y la pérdida de la competencia. La competencia requerida dependerá de la complejidad de las decisiones ${ }^{14}$.

La evaluación de la competencia debe fundarse en ciertas consideraciones. Entre ellas, las demencias no causan una pérdida total de capacidades cognitivas, ni de la capacidad de toma de decisión. La gran mayoría de los síndromes demenciales son de carácter progresivo, afectándose primero las decisiones de mayor complejidad. A mayor conocimiento sobre las demencias, el diagnóstico se establece en etapas más precoces en presencia de déficits cognitivos leves. Un ejemplo es la enfermedad de Alzheimer. Por consiguiente, la evaluación de la competencia de una persona con demencia debe considerar, entre otros, el grado de deterioro cognitivo y la complejidad de la decisión a tomar. En materias de salud, el grado requerido de capacidad es directamente proporcional al nivel de riesgo asociado con una decisión.

En pos de respetar el principio de autonomía, es fundamental optimizar el diagnóstico de capacidad funcional en materia de salud ${ }^{14,18}$. Para ello, Simón y Júdez (2005) y Alvaro (2012) proponen seguir ciertos criterios. Entre ellos, uno criterio cardinal es que la incompetencia no se base en el diagnóstico ${ }^{14,18}$. De hecho, si bien la competencia para consentir está relacionada al grado de deterioro cognitivo, instrumentos de evaluación cognitiva como el Mini-Mental Test de Folstein tienen escaso valor predictivo ${ }^{19}$. La evaluación debe ser tarea es- pecífica; la cognición puede fluctuar, la evaluación debe repetirse si es necesario; la decisión tomada, aunque absurda, no constituye un argumento para declarar a un paciente incompetente; evaluar el proceso de toma de decisión (entender y recibir información; implicación para el paciente; decisión razonada y comunicación de la decisión) y no en la elección ${ }^{20}$. Estos criterios deben considerar el contexto clínico de las personas con demencia, entre los que se encuentran sus trastornos de comunicación verbal, que pueden incitar a minimizar las reales competencias de los pacientes si no se usan métodos de comunicación no verbales y los trastornos en la conciencia de enfermedad. Es común que personas con demencias presenten un déficit en la percepción de sus trastornos o anosognosia, que puede interferir con la toma de decisiones en materias salud. Es decir, no contextualizan la decisión a tomar a su situación, porque no reconocen los efectos de su enfermedad y, por ende, sus decisiones no integran el hecho de tener demencia. Por ejemplo, no internalizan que han perdido las habilidades para conducir un vehículo o gestionar sus finanzas. Es importante recalcar que la presencia de anosognosia no se presenta en todos los pacientes con demencias ni en todas las etapas, ni tiene estricta relación con la progresión de la enfermedad.

Se han desarrollados diferentes instrumentos para evaluar si las competencias necesarias para una de toma de decisión racional están preservadas (Tabla 1). Estos, generalmente, evalúan si las capacidades de las personas cumplen los requisitos señalado en la Tabla 2.

En resumen, las demencias pueden o no causar

Tabla 1. Principales instrumentos para evaluar la capacidad de toma de decisión en personas con demencia (adaptado de Alvaro 2002) ${ }^{14}$

\begin{tabular}{|lll|}
\hline Guía o protocolo & Nombre & Tipo de instrumento \\
\hline Competencia de consentir & White's criteria & Estructura semi-estructurada \\
\hline Aid to Capacity Evaluation & ACE & Estructura semi-estructurada \\
\hline Capacity Assessment Tool & CAT & Estructura semi-estructurada \\
Medida de capacidad de decisión (Decision assessment Measure) & CCTI & Estructura semi-estructurada \\
\hline Hopemont Capacity Assessment Interview & HCAl & Estructura semi-estructurada \\
\hline MacArthur Competence Assessment Tool & MacCAT-T & Estructura semi-estructurada \\
\hline MacArthur Competency Assessment Tool for Clinical Research & MacCAT-CR & Estructura semi-estructurada \\
MacArthur Competency Assessment Tool for Clinical Research & MacCAT-CA & Estructura semi-estructurada \\
\hline
\end{tabular}


Tabla 2. Criterios para evaluar la competencia en materias de salud según los criterios de competencia de White (1994) (40) (adaptado de Slachevsky, Fuentes et al. (2007) ${ }^{35}$ \& Alvaro (2012) ${ }^{14}$

\begin{tabular}{|c|c|}
\hline Criterios & ¿Como evaluarlo? \\
\hline Criterio de informatibilidad & $\begin{array}{l}\text { ¿Puede el paciente con demencia entender lo que se está discutiendo, por } \\
\text { ejemplo, entiende de qué se trata una determinada operación o lo que signi- } \\
\text { fica realmente vivir solo? }\end{array}$ \\
\hline \multirow[t]{2}{*}{ Capacidades cognitivas y afectivas } & $\begin{array}{l}\text { ¿Puede el paciente mencionar o enumerar las diferentes alternativas } \\
\text { existentes? }\end{array}$ \\
\hline & $\begin{array}{l}\text { ¿Puede el paciente señalar cuál es el más probable resultado de su opción } \\
\text { o los resultados de cada una de las opciones principales, por ejemplo, "el } \\
\text { doctor dice que yo probablemente moriré si no me practico esta operación"? }\end{array}$ \\
\hline \multirow[t]{3}{*}{ Capacidad de elegir } & $\begin{array}{l}\text { ¿Puede la persona decidir entre las diferentes opciones e indicar la que } \\
\text { prefiere? }\end{array}$ \\
\hline & ¿Puede la persona justificar su elección? \\
\hline & ¿Mantiene a través del tiempo su decisión? \\
\hline Capacidad de entender la toma de decisión & Capacidad de explicar los procesos de la toma de decisión \\
\hline
\end{tabular}

una pérdida de competencia en tarea específica, lo que se contrapone con la normativa chilena, que se revisa a continuación.

\section{Chile. Capacidad jurídica y toma de decisiones en salud}

La primera observación a realizar es que la doctrina y jurisprudencia chilenas son concordes en sostener que la demencia debe ser interpretada en sentido amplio ${ }^{21,22}$, es decir, la expresión demente se extiende a todo tipo de enajenación mental o alteración que prive de razón a un individuo, sin importar el nombre científico de la enfermedad que lo produce; ello ha sido tradicionalmente interpretado así, desde la entrada en vigencia del código civil en 1857 (período en que la esperanza de vida al nacer en Chile no superaba los 30 años de edad $)^{23}$.

Por ello, el vocablo "demente" que utiliza el código civil (artículos 456 a 468) no se comprende en sentido técnico, de acuerdo con el significado que le asigna psiquiatría o neurología, sino en su sentido etimológico (locura, trastorno de razón [Real Academia Española] $)^{24}$ y, en general, en los casos en que las personas viven con alguna enfermedad mental que les impida tomar decisiones libremente. Las personas declaradas judicialmente en interdicción por demencia son consideradas absolutamente incapaces (artículo 1.447); se entiende que carecen de voluntad, de suficiente juicio o discernimiento y no pueden ejecutar acto jurídico alguno por sí mismos, entre otros: testar, reconocer un hijo, celebrar contratos y votar ${ }^{25}$. Uno de los fines históricos que explica la restricción enunciada es la protección del patrimonio y su administración. Los actos o contratos del demente posteriores al decreto judicial de interdicción son considerados nulos, aunque se alegue haberlos celebrado o ejecutado en un intervalo lúcido (artículo 465 código civil de la República de Chile). Una vez declarado interdicto, el demente podría llegar a ser rehabilitado en su capacidad, a través de nuevas gestiones judiciales que dan lugar a una sentencia de rehabilitación que pone término a la interdicción ${ }^{26}$.

Con relación a lo descrito en el párrafo precedente, la Ley No 18.600 que establece normas sobre deficientes mentales, define persona con "discapacidad mental" como aquella que a consecuencia de una o más limitaciones psíquicas, congénitas o adquiridas, previsiblemente de carácter permanente $y$ con independencia de la causa que las hubiera originado, vea obstaculizada, en o a lo menos un tercio, su capacidad educativa, laboral o de integración social (artículo 2 Ley No 18.600). Sostiene que la persona con discapacidad mental grave y profunda permanecerá al cuidado de su familia, no obstante, habrá establecimientos especiales en caso de que el propio hogar no les cobije, que funcionarán bajo la tuición del Ministerio de Salud y de Justicia. La 
atención de personas con discapacidad mental grave o profunda tendrá como objeto fundamental lograr su integración a la sociedad (artículo 9 Ley No 18.600).

Por otro lado, y según prescribe la Ley No 20.422 (2010) que establece normas sobre igualdad de oportunidades e inclusión social de personas con discapacidad, la discapacidad mental la declara administrativamente la Comisión de Medicina Preventiva e Invalidez -COMPIN- dependientes del Ministerio de Salud (MINSAL) o las instituciones públicas o privadas, reconocidas para estos efectos por el MINSAL (artículo 13). La declaración de discapacidad mental que realizan estas instituciones tiene por finalidad permitir a los beneficiarios acceder a apoyos sociales y legales. Es una resolución que emana de un órgano administrativo sin facultad para afectar el ejercicio de autonomía de una persona, como, por ejemplo, declararla interdicta. La declaración administrativa de discapacidad puede ser sujeta a un procedimiento de recalificación por la COMPIN, previa solicitud fundada del interesado. No podrá solicitarse la recalificación más de una vez en cada año calendario, a menos que esta solicitud se fundare en hechos o antecedentes nuevos, no vinculados a las circunstancias que dieron lugar a la calificación (artículo 14).

Por consiguiente, la "demencia" a la que alude el código civil y "discapacidad mental" a la que alude las leyes en comento, no son sinónimos. La declaración administrativa de discapacidad mental puede constituir un paso previo a la sentencia judicial que declara la interdicción por causa de demencia de una persona. Pero, el único facultado para afectar derechos humanos por falta de autonomía es el juez, previo pronunciamiento judicial. La interdicción por demencia es gravosa porque el interdicto queda anulado de su participación en la vida civil ${ }^{27,28}$.

La situación normativa en esta materia da cuenta de la influencia que ha tenido en Chile (y en países latinoamericanos) la tradición del derecho europeo continental (de base romana, asumido en el proceso de codificación francés), que considera como modelo de toma de decisiones aquel que se basa en la "sustitución de la voluntad" de la persona jurídicamente incapaz por aquella voluntad de un representante capaz de representarlo y, que se asume refleja el interés superior del representado (al menos como ficción jurídica ${ }^{29}$.

\section{Discusión}

El ordenamiento jurídico presenta serias limitaciones. Por una parte, no es acorde a la complejidad clínica de las demencias que producen un compromiso gradual de las competencias y por otra, es anacrónico. El régimen que propone el código de antaño parece al juicio de hoy como fruto de un paternalismo jurídico, y también médico, cuestionable, precisamente porque la voluntad y voz del representante no necesariamente es correlativo a los intereses legítimos del representado o su intención real.

Finalmente, el orden público internacional avanza hacia el modelo de toma de decisiones basado en el "apoyo o a asistencia de la voluntad", que busca la igualdad de las personas con discapacidad en el ejercicio de su capacidad jurídica ${ }^{30}$. Así lo consagra el artículo 12 No 2 de la Convención Internacional sobre Derechos de las Personas con Discapacidad (2006) que es vinculante para Chile desde el año 2008, y prescribe que el igual reconocimiento ante la ley y de la personalidad jurídica en las personas con discapacidad. El documento define también la "discriminación por motivos de discapacidad" que incluye expresamente la denegación de "ajustes razonables", entendidos como las modificaciones y adaptaciones necesarias y adecuadas que no impongan una carga desproporcionada o indebida, cuando se requieran en un caso particular, para garantizar a las personas con discapacidad el goce o ejercicio de sus derechos $y$ libertades (artículo 2 incisos $4^{\circ}$ y $5^{\circ}$ ).

Por lo anterior, en pos del bien común y del respeto de derechos humanos, es necesario repensar el ordenamiento chileno para superar la asincronía entre la competencia en la toma de decisiones y la capacidad jurídica, su anacronismo y su inadecuación a la normativa internacional vigente y vinculante. Los nuevos instrumentos jurídicos deben ser concordantes con las realidades clínicas y de investigación, sin desconocer el proceso de toma de decisión en los trastornos demenciantes. Entre otras cosas, se debe considerar que las demencias pueden comprometer la toma de decisión selectivamente en algunos dominios específicos. Adicionalmente, se debe distinguir entre discapacidad y dependencia, esta última considera la necesidad de otra persona para hacer las actividades de la vida diaria, sean estas instrumentales o básicas. 
Estos cambios de paradigma que apuntan a la inclusión efectiva se están implementando paulatinamente en países de la Organización para la Cooperación y el Desarrollo Económico (OCDE) como Irlanda, Italia y Francia ${ }^{31,32}$. Una propuesta es reformar el sistema de protección de las personas jurídicamente incapaces, sustituyendo la expresión "demente" por personas con disminución de sus facultades psíquicas (personas con autonomía disminuida, personas privadas de

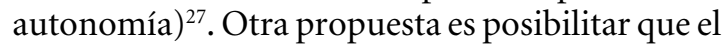
juez adecue las limitaciones a la autonomía de la persona incapaz, según el grado de autodirección que presente. En cualquier caso, las propuestas deben considerar un criterio de gradualidad de la capacidad jurídica y su revisión judicial permanente (en tribunales especializados) ${ }^{33}$.

\section{Referencias}

1. Prince M, Bryce R, Albanese E, Wimo A, Ribeiro W, Ferri CP. The global prevalence of dementia: a systematic review and metaanalysis. Alzheimers Dement 2013; 9 (1): 63-75 e2.

2. Mackenbach JP, Karanikolos M, Looman CW. The rise of mortality from mental and neurological diseases in Europe, 1979-2009: observational study. BMC public health $2014 ; 14: 840$.

3. Slachevsky A, Arriagada P, Maturana J, Rojas R. Enfermedad de Alzheimer y otras Demencias en Chile: Propuesta de un Plan Nacional de Alzheimer y otras demencias. CopradSonepsyn, 2012.http://www.coprad. cl/coprad/wp-content/uploads/2012/12/Resumen_ejecutivo.pdf

4. Slachevsky A, Abusleme Lama M, Arenas Massa A. Cuidados paliativos en personas con demencia severa: reflexiones y desafios. Rev Med Chile 2016; 144: 94-101.

5. Arenas Massa A. Adulto mayor, nuevas perspectivas para el desarrollo humano. Santiago: Ediciones Universidad Finis Terrae 2012. 202 p.

6. D'Agostino F. Introduzione alla biopolitica, Dodici voci fondamentali. 1 ed. Roma: Aracne Editrice; 2009. 205 p.

7. Sgrecia E. Manuale di Bioetica. 3 ed. Milani: Vita \& Pensiero; 2003. 1016 p.

8. Thagard P. The Moral Psychology of Conflicts of Interest: Insights from Affective Neuroscience. Journal of Applied Philosophy 2007; 24 (4): 367-80.

9. De Neys W. Heuristic Bias, Conflict, and Rationality in Decision-Making. In: Glatzeder B, Goel V, von Müller A, editors. Towards a Theory of Thinking Building Blocks for a Conceptual Framework. Berlin, Heidelberg: Springer-Verlag 2010. p. 23-33.

10. Lidz CW, Appelbaum PS, Grisso T, Renaud M. Therapeutic misconception and the appreciation of risks in clinical trials. Social science \& medicine 2004; 58 (9): 1689-97.

11. Henderson GE, Churchill LR, Davis AM, Easter MM, Grady C, Joffe S, et al. Clinical trials and medical care: defining the therapeutic misconception. PLoS medicine. 2007; 4 (11): e324.

12. Kahneman D, Frederick S. Frames and brains: elicitation and control of response tendencies. Trends in cognitive sciences 2007; 11 (2): 45-6.

13. Sharot T, Riccardi AM, Raio CM, Phelps EA. Neural mechanisms mediating optimism bias. Nature 2007; 450 (7166): 102-5.

14. Alvaro LC. [Competency: general principles and applicability in dementia]. Neurologia 2012; 27 (5): 290-300.

15. Clarke A. The Neuroscience of Decision Making and Our Standards for Assessing Competence to Consent. Neuroethics. 2013; 6: 189-96.

16. Welie JVM, Welie SPK. Patient Decision-Making Competence: Outline of a Conceptual Analysis. Health Care and Philosophy. 2001; 4: 127-38.

17. Radoilska L. Introduction: personal autonomy, decisional capacity and mental disorder. In: Radoilska L, editor. Autonomy and Mental disorder. Oxford: Oxford University Press; 2012. p. ix-xli.

18. Simón P, Júdez J. Consentimiento informado. In: Gracia D, Júdez J, editors. Bioética para clínicos. Madrid: Editorial Triacastela; 2005. p. 33-54.

19. Warner J, McCarney R, Griffin M, Hill K, Fisher P. Participation in dementia research: rates and correlates of capacity to give informed consent. Journal of medical ethics 2008; 34 (3): 167-70.

20. Trachsel M, Hermann H, Biller-Andorno N. Cognitive fluctuations as a challenge for the assessment of decision-making capacity in patients with dementia. Am J Alzheimer dis Other dem 2015; 30 (4): 360-3.

21. Corte de Concepción, 10 de junio de 2008, rol N ${ }^{\circ} 240$ 2008. Concepción: Base de Jurisprudencia de Legal Publishing $\mathrm{N}^{\circ}$ 39216. 2008.

22. Corte de Concepción 27 agosto 1896, t. II, No 3.325 . 1896.

23. Gutiérrez Roldán H. La población en Chile, World Population Year 1974. Paris: CICRED; 1975. 86 p.

24. Diccionario de la Real Academia de la Lengua Española. Madrid: Real Academia de la Lengua Española; 2014.

25. Larraín H. Lecciones de Derecho Civil. Santiago: Ed. Jurídica de Chile; 1994. 503 p.

26. Claro Solar L. De la sucesión por causa de muerte (t.13- 
15). Explicaciones de derecho civil chileno y comparado. 8. Santiago: Ed. Jurídica de Chile; 1978. p. 22-3.

27. Corral H. Interdicción de personas que sufren trastorno de dependencia a la cocaína. Revista de Derecho. 2011; XXIV: 31-64.

28. Vial del Río A. Teoría General del Acto Jurídico. Santiago: Ed. Jurídica de Chile; 2003-2007. 407 p.

29. Talamanca MI. Instituzioni di Diritto Romano. Milano: Giuffrè Editori; 1990. 829 p.

30. A. P. El modelo social de la discapacidad. Orígenes, caracterización y plasmación en la Convención Internacional sobre los derechos de las personas con discapacidad. Madrid: CINCA; 2008. 526 p.

31. WHO. Dementia: a public health priority. Report.
World Health Organization (WHO) Alzheimer's Disease International, 2012 2012. Report No.

32. Principios para la protección de los enfermos mentales y el mejoramiento de la atención de la salud mental. Washington: Organización de Naciones Unidas, 1991.

33. Dementia: autonomy and decision-making Putting principles into practice. Scotland: Alzheimer Scotland, 2013.

34. White B. Competence to consent. Washington: Georgetown University Press; 1994.

35. Slachevsky A, Fuentes P, Javet L, Alegría P. Enfermedad de Alzheimer y Otras Demencias. Guía para Familiares y cuidadores. Santiago, Chile: Editorial LOM; 2007.

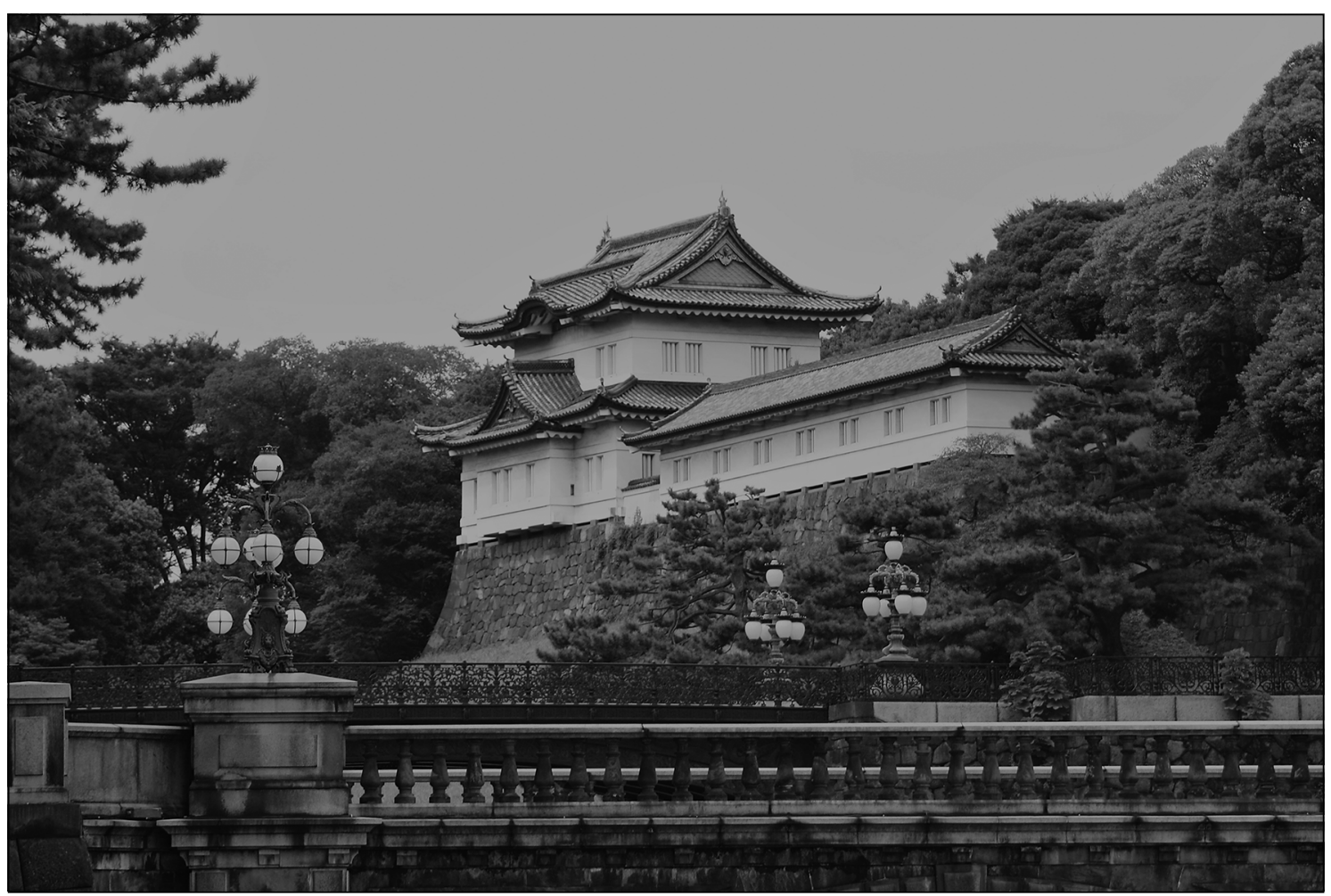

Palacio Imperial. Tokio. Dr. Joaquín Palma Heldt 